\title{
Primary Cardiac Rhabdomyosarcoma in a child - A case report
}

\author{
KRISHNA PRASAD MARAM ${ }^{1}$, Vikram Kudumula ${ }^{2}$, and Dilip Ratti ${ }^{2}$ \\ ${ }^{1}$ Affiliation not available \\ ${ }^{2}$ Andhra Hospitals
}

November 30, 2020

\begin{abstract}
Primary cardiac tumors are rare in children, usually consist of benign tumors like rhabdomyomas and fibromas that may spontaneously regress. Primary malignant tumors are extremely rare even in adults and very few paediatric cases were reported in literature. Rhabdomyosarcoma is a rare primary malignant tumor in children and most of the reported cases occur in right ventricle, left atrium and right atrium. We report a 15 month old child with primary rhabdomyosarcoma of left ventricle presenting in cardiac tamponade and circulatory failure.
\end{abstract}

\section{Introduction:}

Cardiac tumors in children are rare, with the majority being benign primary tumors like rhabdomyoma and fibroma.Malignant tumors of the heart are extremely rare in children, with the majority being sarcomas followed by lymphomas.However, unlike adults, metastatic cardiac tumors are less common than primary malignant tumors of the heart in children. Children with cardiac tumors can be asymptomatic or may present with chest pain, murmur, arrhythmia, heart failure, or sudden death, depending on the location and size of the tumor [1]. We report a very rare case of primary rhabdomyosarcoma of the heart presenting with a life threatening pericardial effusion, and massive local tissue infiltration.

\section{Case Report}

A 15-months old boy had presented with fever and respiratory distress of three days duration. At the time of admission, he had signs and symptoms of acute circulatory failure with tachypnea, tachycardia, and hypotension. He was febrile and irritable, and had no rash. Chest auscultation showed good bilateral air entry but muffled heart sounds. Abdominal examination was otherwise normal except for hepatomegaly.He had no significant history of illness prior to the current admission. He had normal growth and vaccinated as per recommended schedule. His CXR demonstrated significant cardiomegaly and a bedside 2D-ECHO (Fig.1) showed a massive pericardial effusion causing an impending tamponade, and a solid vascular mass in the left ventricle extending into the anterior as well as superior mediastinum. In view of hemodynamic instability, he underwent an immediate pericardiocentesis which drained around $200 \mathrm{ml}$ of blood stained fluid. A smear study of the pericardial fluid showed benign reactive mesothelial cells, macrophages, neutrophils but had no evidence of malignancy. In light of 2D ECHO findings, it was decided to do a surgical evaluation of the cardiac mass. 


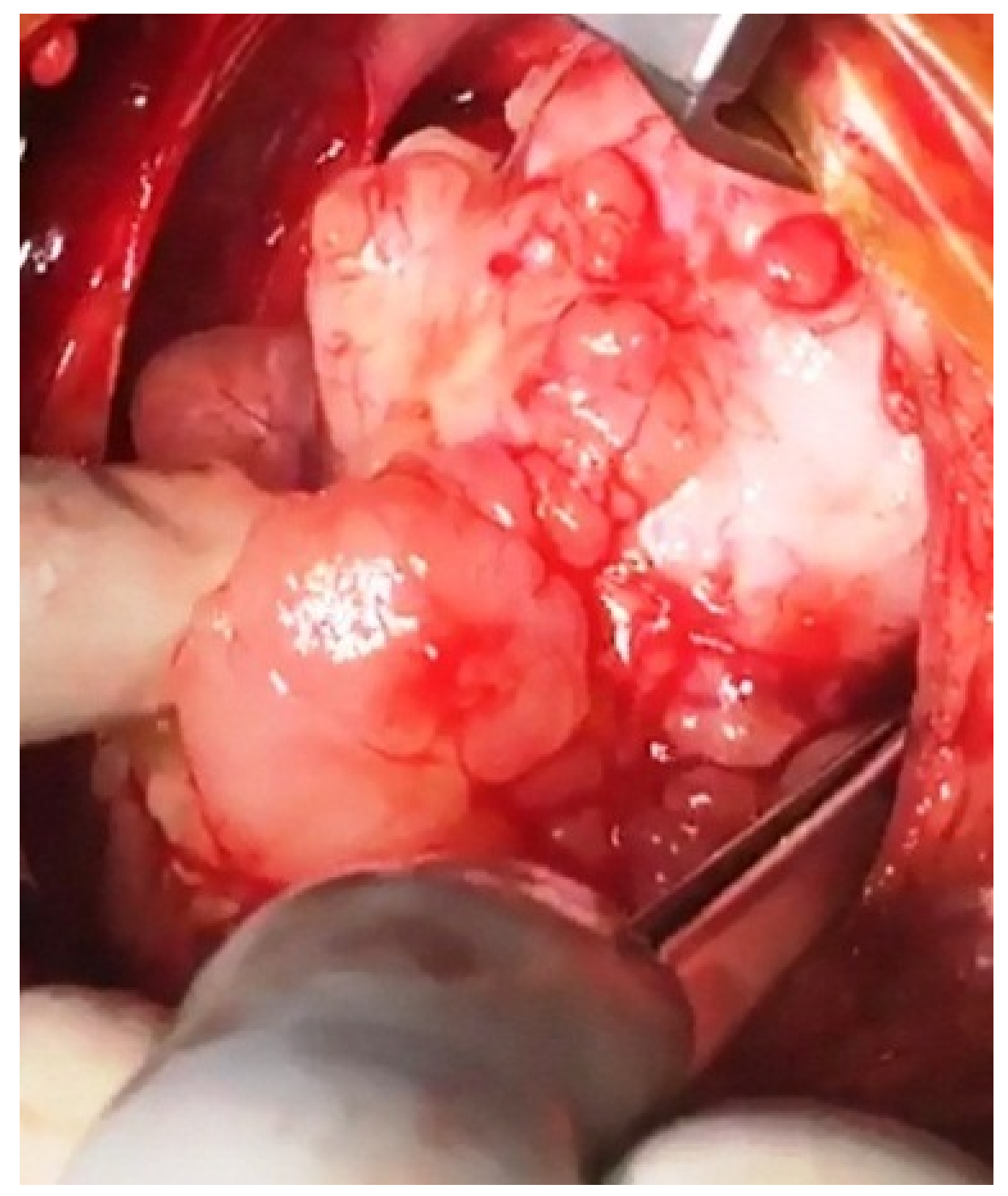




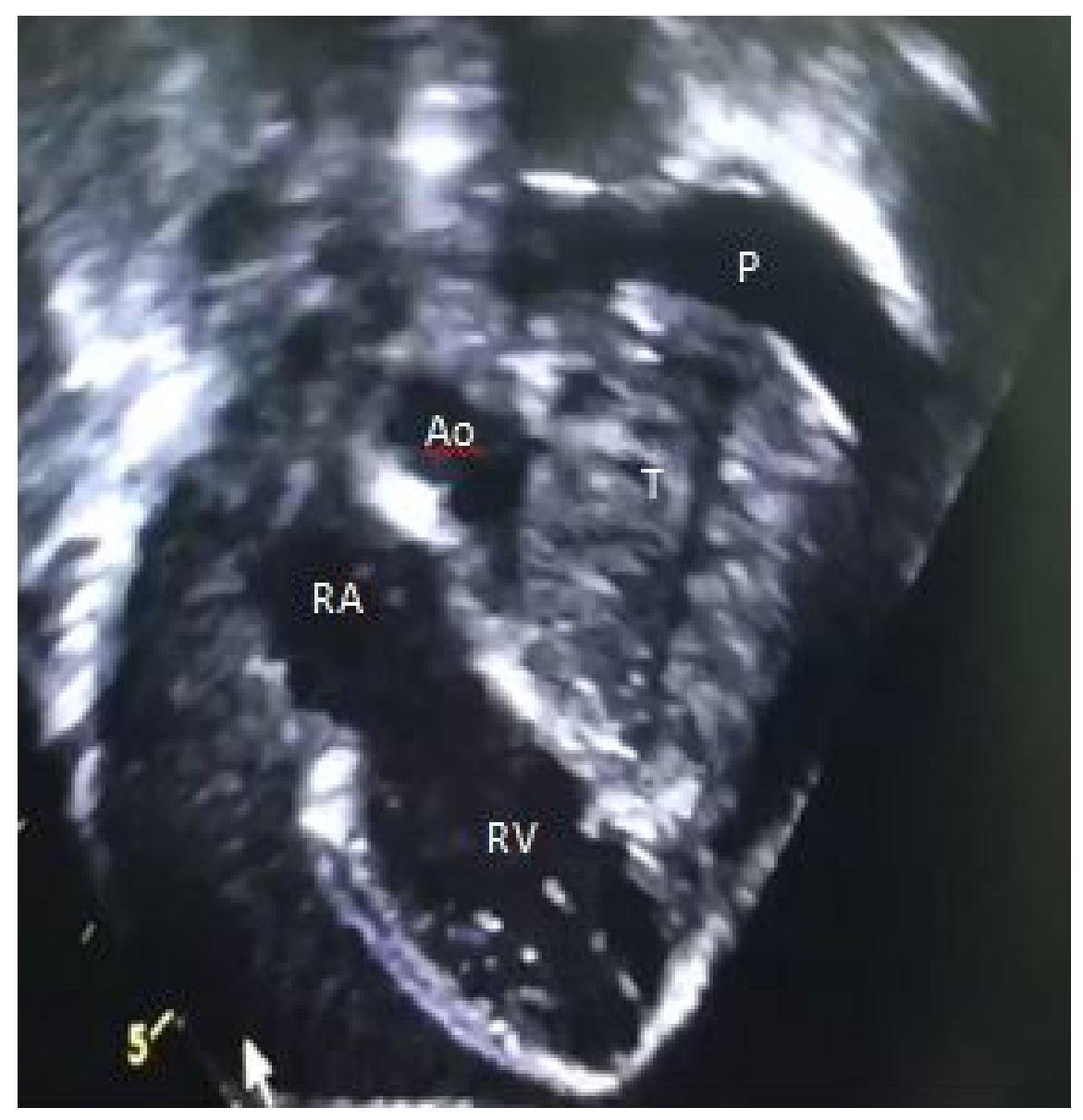

A midline sternotomy revealed a large lobular mass (Fig 1) arising out of the left ventricle, infiltrating the myocardium and extending into the anterior and superior mediastinum. Due to the extent of tumor infiltration, it was considered inoperable. The biopsy showed a tumor with intermingled dense, compact cellular and loose myxoid areas. The cellular areas are predominantly seen around dilated blood vessels, and composed of sheets of small spindled to round cells with hyperchromatic, round nuclei. The biopsy and immunohistochemistry analysis were consistent with a diagnosis of spindle cell rhabdomyosarcoma.

\section{Discussion}

Primary malignant tumors of the heart are extremely rare, constituting around $10 \%$ of cardiac tumors in children. Sarcomas are the most common primary cardiac malignant tumors (95\%) followed by lymphomas [1].Angiosarcoma, rhabdomyosarcoma and undifferentiated sarcomas are the most common sarcomas in children in the order of frequency of occurrence [2].

Rhabdomyosarcomas are mesenchymal tumors originating from undifferentiated striated muscle [1]. They are more common in males [3] and show extensive and aggressive local tissue infiltration and vessel extension [4]. Most lesions tend to be single and occur more frequently in the left atrium but, they can involve any cardiac chamber. Histologically, they are classified as embryonal, alveolar, pleomorphic and other types while embryonal variety $(75 \%)$ is the most common type in pediatric population [5]. Most cases of rhabdomyosarcomas are sporadic in nature although association with familial syndromes like LiFraumeni and Neurofibromatosis type I is not uncommon [6]. They usually present with signs and symptoms congestive heart failure, chest pain, outflow tract obstruction, recurrent pericardial effusions, arrhythmias, pulmonary emboli and rarely sudden death depending upon the size and location of the tumor. Echocardiography or Computing 
Tomography/MRI of the heart helps with establishing the diagnosis. Open surgical or endomyocardial biopsy aids the histological diagnosis of the tumor. A thorough search with CT/MRI must be made to ascertain the metastatic spread of the lesion. Palliative debulking surgery, chemotherapy and radiotherapy extend survival but prognosis is extremely poor with short survival (upto 13 months) due to local infiltration and metastasis [7]

\section{References:}

1. Uzun O, Orhan Uzun,Wilson,DG, Vujanic GM,Parsons JM, De Giovanni JV. Cardiac tumors in children. Orphanet Journal of Rare Diseases.2007; 2:11:1-14.

2. Tao TY,Yahyavi-Firouz-Abadi N, Singh GK, Bhalla S. Pediatric Cardiac Tumors: Clinical and Imaging Features. RadioGraphics. 2014;34(4):1031-1046.

3. Roberts WC.Primary and secondary neoplasms of the heart.Am J Cardiol.1997; 80:671-682.

4. Nadas AS, Ellison RC.Cardiac tumors in infancy. Am J Cardiol.1968; 21:363-366.

5. Ota S, Shimonaga T,Yuki S, Kuraoka K, Ogawa T, Hirata T.Eribulin therapy for primary cardiac rhabdomyosarcoma. Anticancer Drugs. 2020;31(3):304-309.

6. Dasgupta R, Fuchs J Rodeberg D. Rhabdomyosarcoma. Seminars in Pediatric Surgery.2016; 25(5):276283.

7. Burke A, Virmani R. Pediatric heart tumors. Cardiovascular Pathology.2008; 17(4):193-198.

\section{Declarations:}

Ethics approval and consent to participate: Not Applicable

Consent for publication: Not applicable

Availability of data and materials : Yes

Competing interests: None

Funding : None

Authors' contributions: Dr Krishna Prasad and Dr Vikram is responsible for manuscript preparation and data collection.Dr Dilip is involved in case management.

Acknowledgements: None 